\title{
Validation of Parboiling Rice Quality Simulation Mathematical Model
}

Gunathialke DMCC*

Department of Soil Science \& Agricultural Engineering, College of Agriculture, Fisheries and Forestry, Fiji National University, Fiji

\begin{abstract}
Parboiling (hydrothermal treatment) of paddy/rough rice is an ancient traditional process of South Asian countries and it's reducing the level of grain breakage and increase in head yield of rice during milling. However, parboiling of rice associated minor drawbacks such as reduce rice kernel whiteness and increase in kernel hardness. Level of grain breakage, kernel whiteness and hardness can be altered by parboiling treatments such as water temperature and duration of soaking. The mathematical model developed by Gunathilake can be used to predict the rice quality values such as kernel whiteness, harness and broken grains however, model should be validated (verified) before applied. Hence, this research study was carried out to validate the mathematical model for checking its suitability and accuracy to predict above mentioned rice quality values in local rice variety. BG 358 short grain rice was used for validation/verification the model. In this model single paddy grain parboiling (hydration) was considered. Results were shown that mathematical model is fit for predict rice quality values of kernel whiteness, hardness, broken grain percentage and head rice yield percentage, by the function of water temperature and duration of soaking are the major treatments of paddy parboiling. Goodness of fit $(E)$ value of actual values and predicted values of kernel whiteness, hardness broken grain percentage and head rice yield were showed 5.91, 6.73, 8.32 and 7.92 respectively. The average Goodness of fit $(E)$ value was 7.22. Hence, it was revealed, that the mathematical model was capable to predict rice quality values by water temperature and soaking duration similar to actual values of rice qualities. Hence, it can be concluded that mathematical model was good fit to simulate above rice qualities by the paddy parboiling treatments of water temperature and soaking duration for BG 358 paddy.
\end{abstract}

Keywords: Paddy Parboiling; Mathematical model; Model validation; Rice quality; Simulation; Prediction

\section{Introduction}

Parboiling of rice is a traditional process in Southern Asia since ancient time. Generally, parboiling process consists of three stages: soaking the cleaned raw rough rice to saturation moisture content, gelatinization of rice starch by adding heat to the moist kernels through steaming, and drying the product to moisture content suitable for milling or storage. Water and heat are the two main elements in the hydro-thermal process. It can be achieved through a variety of methods that differ basically in the intensity of the hydrothermal treatment water temperature soaking and steaming duration/time [1,2]. Parboiling causes physical and chemical modifications in the grain, leading to favorable changes such as easier

shelling, higher head rice yield, fewer broken grains, increased resistance to insects, less solids loss during cooking, better retention of nutrients (e.g. vitamins, and minerals), and higher oil content in the bran [1-3]. It has been established that cracks, chalkiness, and incomplete grain filling are totally rectified and many of the previous defects are cured when paddy is parboiled properly [1]. Although parboiled rice has been reported lot of advantage, parboiling process caused some drawbacks such as loss aromatic value, taste, colour, texture and change of palatability characteristics and also it constitute additional expenditure in rice processing $[1,2]$.

The mathematical model developed by Gunathilake [4] for simulation of paddy quality as a function of parboiling process can be used to predict the values of rice qualities such as kernel whiteness, broken grain $\%$, head rice yield $\%$ and kernel hardness by the function of parboiling treatments of water temperature and duration of soaking. However, the predicted values by mathematical model should be validated with actual values for verification its suitability to predict rice quality values. This mathematical model has been validated/verified with above mentioned actual rice quality values that obtained by parboiling of BG 358 (Samba) paddy verity with predicted values obtained by mathematical model. Goodness of fit parameter was adopted to evaluate the capability of mathematical expression to predict the data. Goodness of fit value below $10 \%$ are indicative of responsibility good fit, $10-20 \%$ fairly good fit and $20-30 \%$ not satisfactory fit for all purpose. Objective of this study is to validate the predicted values obtained by mathematical model with actual values obtained by parboiling of BG 358 paddy verity.

\section{Materials and Methods}

The mathematical model for rice quality simulation by the function of paddy parboiling treatments

Moisture migration in grains during soaking of paddy is usually assumed to occur by diffusion caused by the moisture gradient between the surface and the centre of grain [5]. Water absorption into the rice kernel can be regarded as a process of diffusion which a proportion of the absorbed water becomes immobilized during water-starch reaction (gelatinization) as water diffusion proceeds [6]. Such reaction will occur if the reactivity and affinity of starch with water and temperature are high enough. The gelatinization temperature varies for different varieties anywhere between $70-85^{\circ} \mathrm{C}$.

To simulate both diffusion and reaction rate phenomena for water on rice grains the following assumptions are made:

*Corresponding author: Gunathialke DMCC, Assistant Professor, Department of Soil Science \& Agricultural Engineering, College of Agriculture, Fisheries and Forestry, Fij National University, Fiji, Tel: (679) 347 9200; E-mail: cchampathi@gmail.com

Received June 25, 2018; Accepted August 10, 2018; Published August 14, 2018

Citation: Gunathialke DMCC (2018) Validation of Parboiling Rice Quality Simulation Mathematical Model. J Food Process Technol 9: 746. doi: 10.4172/21577110.1000746

Copyright: ( 2018 Gunathialke DMCC. This is an open-access article distributed under the terms of the Creative Commons Attribution License, which permits unrestricted use, distribution, and reproduction in any medium, provided the original author and source are credited. 
Citation: Gunathialke DMCC (2018) Validation of Parboiling Rice Quality Simulation Mathematical Model. J Food Process Technol 9: 746. doi: 10.4172/2157-7110.1000746

Page 2 of 5

a. The process is considered to be a simultaneous unsteadystate water diffusion and first-order irreversible water-starch reaction.

b. The diffusion coefficient and reaction rate constant are independent of moisture content.

c. There is no change of grain volume during water absorption.

d. Mass transfer coefficient is high enough to assure constant moisture content at the surface of the grain since the beginning of absorption process.

e. Thermal effects are neglected, i.e. grain temperature is assumed uniform and equal to the water temperature.

f. The shape of paddy grains is assumed a sphere and radius $\left(r_{e}\right)$ of the paddy grain was calculated, width of the grain divided by two.

g. Increase in kernel moisture content due to steaming is neglected in comparison to moisture increase by soaking.

h. Hydration (water absorbent) of single paddy grain was considered in this simulation because; the quality traits of a single grain kernel can produce an index of the overall quality of the bulk grain, as individual kernels comprise a grain bulk. Therefore, single-kernel hydration behavior is important in understanding the overall quality of grain.

The differential equation expressing simultaneous water diffusion and reaction process in a solid sphere is [7]:

$$
\frac{\partial C}{\partial t}=D_{\mathrm{f}}\left(\frac{\partial^{2} C}{\partial r^{2}}+\frac{2}{r} \frac{\partial C}{\partial r}\right)-k C
$$

Where $\mathrm{C}$ is moisture content, $\mathrm{t}$ is the time, $\mathrm{r}$ the radius and $\mathrm{D}$ and $\mathrm{k}$ are the effective diffusion coefficient and reaction rate constant, respectively. To integrate Equation 1 the following boundary and initial conditions were used:

$$
\begin{aligned}
& t>0 \text { and } r=0, \partial C / \partial r=0 \\
& t=0 \text { and } 0 \leq r \leq r_{e}, C=C_{0} \\
& t>0 \text { and } r=r_{e}, C=C_{s}
\end{aligned}
$$

Where $r_{e}$ is the radius of the paddy grain and $C_{s}$ and $C_{o}$ are the saturation and initial moisture content, respectively. The integration of Equation (1) and Equation (2)-(4) allows to obtain the total amount of water absorbed as a function of time which, expressed in terms of the instantaneous moisture content is [7]

$$
m=m_{0}+\left(m_{0}-m_{s}\right) 8 \pi r_{e} D_{\mathrm{f}} C_{0} \times \sum_{n=1}^{\infty}\left\{\frac{A_{n} k r_{e}^{2} t-B_{n} r_{e}^{2} \exp \left(-\left(A_{n} t / r_{e}^{2}\right)\right)}{A_{n}^{2}}\right\}
$$

$B_{n}=n^{2} \pi^{2} D_{\mathrm{f}}$

$A_{n}=k r_{e}^{2}+D_{\mathrm{f}} n^{2} \pi^{2}$ Where

$\mathrm{m}=\mathrm{absorbed}$ instantaneous moisture content

$\mathrm{m}_{\mathrm{s}}=$ Saturation moisture content

$\mathrm{m}_{\mathrm{o}}=$ Initial moisture content

$\mathrm{C}_{\mathrm{o}}=$ Equilibrium initial moisture content

$r_{e}=$ radius of the paddy grain from the breadth $\mathrm{n}=$ Number of paddy grain $(\mathrm{n}=1)$

$\mathrm{t}=$ Time

Diffusion coefficient $\left(D_{f}\right)$ and reaction rate constant $(k)$ are depend on soaking water temperature. Following values for paddy hydration was determined by Bellow et al. [8].

$$
\begin{aligned}
& \mathrm{D}_{\mathrm{f}} \text { at } 25^{\circ} \mathrm{C}=1.40 \times 10^{-11} \text { and } \mathrm{D}_{\mathrm{f}} \text { at } 70^{\circ} \mathrm{C}=7.17 \times 10^{-11} \\
& \mathrm{k} \text { at } 25^{\circ} \mathrm{C}=2.29 \times 10^{-10} \text { and } \mathrm{k} \text { at } 70^{\circ} \mathrm{C}=3.50 \times 10^{-5}
\end{aligned}
$$

Amount of moisture content increase in paddy kernel during soaking is also very important to keep up desired quality of rice. Nonlinear regression equations was developed to obtain relationships between the rice quality values i.e. kernel whiteness, broken grain \%, head rice yield $\%$ and kernel hardness and amount of moisture increase in paddy kernel under different soaking conditions of paddy (water temperature and duration of soaking). Following equations (8), (9), (10) and (11) were shown the relationships between rice qualities and paddy kernel moisture content (Effect of paddy kernel moisture content for altering the rice quality). Basmati 370 paddy variety hydration was used for obtaining the following equations [4].

\section{Nomenclature}

Wh Rice kernel whiteness $S c$ Soaking water temperature

$\mathrm{Br}$ Broken kernel percentage $S t$ Soaking duration (time)

$H y$ Head rice yield percentage $S p$ Steaming pressure

$H d$ Rice kernel hardness $S d$ Steaming duration (time)

$M C$ Rice kernel moisture content (wet basis) $R^{2}$ Correlation coefficients

$$
\begin{aligned}
& \mathrm{Wh}=79.9565-0.891699 \mathrm{MC}+0.00790 \mathrm{MC}^{2}\left(\mathrm{R}^{2}=0.945\right) \\
& \mathrm{Br}=441.381-34.4583 \mathrm{MC}+0.693320 \mathrm{MC}^{2}\left(\mathrm{R}^{2}=0.931\right) \\
& \mathrm{Hy}=-229.330+23.0089 \mathrm{MC}-0.455019 \mathrm{MC}^{2}\left(\mathrm{R}^{2}=0.936\right) \\
& \mathrm{Hd}=252.757-17.6395 \mathrm{MC}+0.529237 \mathrm{MC}^{2}\left(\mathrm{R}^{2}=0.864\right)
\end{aligned}
$$

First derivatives of Equations (8), (9), (10) and (11) were given change of rice qualities values namely kernel whiteness, broken grain $\%$, head rice yield $\%$ and kernel hardness parameters due to the unit absorbent of moisture (wet basis) to the paddy kernel by diffusion during soaking. Equation (12) was pointed out first derivatives of Equation (10) that change of head rice yield due to unit absorbent of moisture content inside to the paddy kernel.

$$
\frac{d H y}{d M C}=23.0089-0.910038 M C
$$

Absorbed moisture content into the rice kernel in dry basis by diffusion during soaking as function of water temperature (at two temperature $25^{\circ} \mathrm{C} \mathrm{\&} 70^{\circ} \mathrm{C}$ ) and time of soaking can be calculated by Equation (5) and then this moisture content convert to wet basis by using Equation (15) and substitute to Equation (12). Accordingly variation (increase or decrease) of head rice yield percentage due to moisture increase by diffusion in paddy kernel as function of water temperature and time of soaking was determined. By adopting same method, amount of variation in other quality characteristics i.e. broken grain percentage, kernel whiteness and hardness were able to predict (based on moisture absorption by rice kernel during soaking treatment as a function of soaking time and temperature of soaking water). Complete paddy hydration during soaking was significantly reduced broken grain percentage in rice milling. Therefore it was clear that 
Citation: Gunathialke DMCC (2018) Validation of Parboiling Rice Quality Simulation Mathematical Model. J Food Process Technol 9: 746. doi: 10.4172/2157-7110.1000746

Page 3 of 5

\begin{tabular}{|c|c|c|c|c|c|}
\hline \multicolumn{3}{|c|}{ Hot water $\left(70 \pm 2^{\circ} \mathrm{C}\right)$ soaked parboiling Treatments } & \multicolumn{3}{|c|}{ Ambient water $\left(28 \pm 3^{\circ} \mathrm{C}\right)$ soaked parboiling Treatments } \\
\hline Treat. No. & Soaking duration (min) & Steaming Duration (min) & Treat. No. & Soaking duration (min) & Steaming Duration (min) \\
\hline 1 & 15 & 6 & 7 & 60 & 6 \\
\hline 2 & 45 & 6 & 8 & 270 & 6 \\
\hline 3 & 75 & 6 & 9 & 520 & 6 \\
\hline 4 & 120 & 6 & 10 & 720 & 6 \\
\hline 5 & 180 & 6 & 11 & 1080 & 6 \\
\hline 6 & 240 & 6 & 12 & 1440 & 6 \\
\hline
\end{tabular}

Table 1: Parboiling treatments performed for validation.

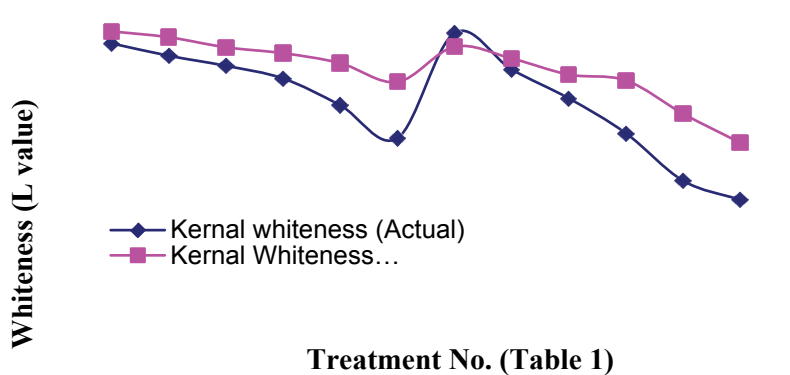

Figure 1: Predicted and actual values of rice kernel whiteness.

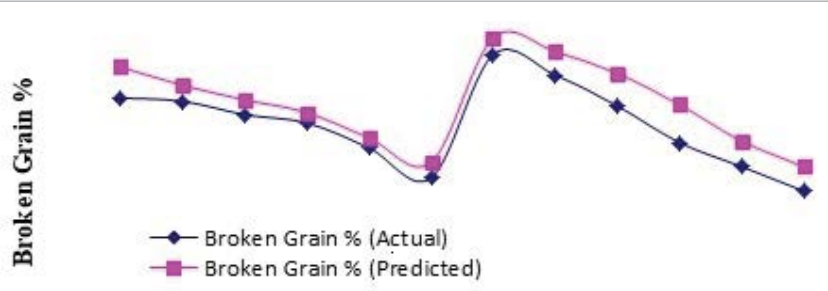

Treatment No. (Table 1)

Figure 2: Predicted and actual values of broken gain percentage.

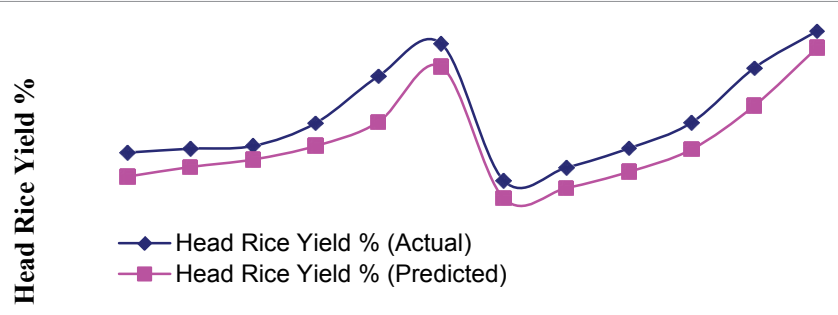

Treatment No. (Table 1)

Figure 3: Predicted and actual values of head rice yield percentage.

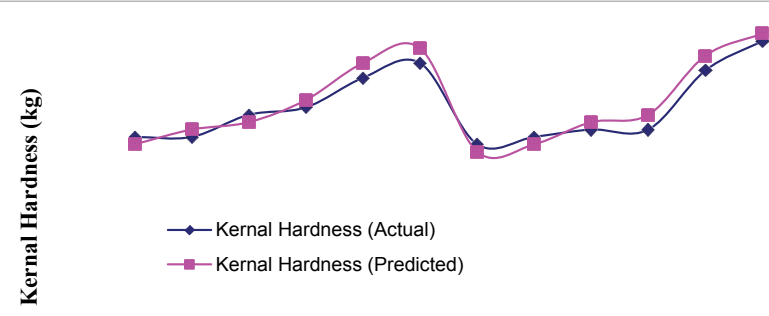

Treatment No. (Table 1)

Figure 4: Predicted and actual values of rice kernel hardness. mentioned rice quality values can be predicted by above mathematical equations based on paddy hydration as function of water temperature and time of soaking in paddy parboiling.

\section{Goodness of fit (E)}

This parameter is widely adopted by many scientists to validate/ verification of the mathematical expressions to check their accuracy and suitability to predict the values. E value below $10 \%$ are indicative of responsibility good fit, $10-20 \%$ fairly good fit and $20-30 \%$ not satisfactory fit for all purpose. E value is calculated by Equation (13)

$$
E=\frac{100}{N} \sum{ }^{n} \frac{\left(R_{\text {actual }}-R_{\text {predicted }}\right)}{R_{\text {actual }}}
$$

BG 358 short grain paddy variety $(\mathrm{L}: \mathrm{B}$ ratio=2.15) which famous among local farmers and consumers was used for model validation. Table 1 is shown the parboiling treatments that carried out for validation the model. The treated paddy samples (soaked different time \& temperature combinations) were dried using laboratory electric dryer until they reach to the moisture content of $14 \%$ (wet basis). When the paddy moisture content reached at $18 \%$ (wet basis) during drying, three hours tempering period was given as method invented, i.e. placing the rice sample in fully covered bin in order to prevent crack formation in rice kernel The dried paddy up to $14 \%$ moisture content was dehulled using the Satake laboratory testing de-husker and the brown (unpolished) rice were polished using the Satake laboratory rice mill (abrasive type polisher). All rice samples were polished for 75 seconds; hence average degree of polish (bran removal) was maintained around 8 percent. Broken grains percentage and head rice yield percentage were calculated using representative working sample of milled rice of $100 \mathrm{~g}$ were obtained using sample divider. Grain partials, which are smaller than the $3 /{ }_{4}$ of the grain, were considered as broken grains and they were separated by hand picking and broken grain percentage was calculated. Accordingly, head rice yield was also calculated. These milling characteristics were obtained using method outlined by Bal et al. [9].

\section{Moisture measurement of the paddy samples (wet basis)}

In static oven drying method, as suggested Association of Official Analytical Chemists [10] was adopted to obtain moisture in paddy for correct drying practices up to $14 \%$ (wet basis). It was measured by drying sample for 24 hours at $120^{\circ} \mathrm{C}$ in static oven drying. Moisture content in the form of wet basis was calculated by using Equation (14)

$$
M_{w b}=\frac{W_{w}}{W_{w}+W_{d m}}
$$

Where,

$\mathrm{M}_{w b}=$ Moisture content (wet basis),

$\mathrm{M}_{d b}=$ Moisture content (dry basis) 
$\mathrm{W}_{w}=$ Weight of water,

$\mathrm{W}_{d m}=$ Weight of the dry matter

To convert percent moisture content from dry basis to wet basis Equation (15) was used

$$
M_{w b}=\frac{100 \times M_{d b}}{100+M_{d b}}
$$

\section{Calculation of rice kernel whiteness and hardness}

Rice kernel whiteness was measured by using Mini-scan XE plus Hunter Lab Colorimeter in Hunter scale L measures lightness (whiteness or darkness). The 'L' value varies from 100 for perfect white and zero (0) for perfect black. L value (lightness value) was used as whiteness value of rice kernel. Compression test was carried out to measure rice kernel hardness. The paddy hardness tester had been adapted to perform a compression test. Force at rupture was considered as the hardness. Three replications were considered for each treatment and the measured force were averaged.

\section{Validation of the parboiling model}

Actual values of rice kernel whiteness, broken grain percentage, head rice yield and rice kernel hardness were obtained by given at two temperatures and 12 different soaking times as show in the Table 1. Predicted values were obtained by given equal temperatures and times as actual treatments. Actual values and predicted values were substituted to Equation (13) and calculated the goodness of fit (E value) of mathematical expressions.

\section{Results and Discussion}

\section{Validation of the model for rice kernel whiteness}

Figure 1 shows the predicted and actual values of rice kernel whiteness with parboiling treatments. The results clearly indicated that predicted values were similar to actual values and it was observed that goodness of fit of the actual values with the predicted values was 5.91. Predicted and actual values of whiteness showed that increases in soaking time of parboiling caused to reduce rice kernel whiteness. Mild parboiling treatment was preserved the kernel whiteness. Model proved that short time of soaking and steaming were preserved the kernel whiteness. Changing of rice kernel whiteness is probably due to gelatinization of the starch and disintegration of the protein bodies in the endosperm with increasing in severity of the hydrothermal treatment of soaking and steaming [11]. The bran and husk pigments might also contribute by diffusing into the endosperm during soaking it can be caused to reduce kernel whiteness.

\section{Validation of the model for broken grain percentage}

Figure 2 shows the predicted and actual values of broken grain percentage with parboiling treatments. The results are shown that the predicted value has similar pattern in comparison to the actual value. Goodness of fit of actual values with predicted values of broken grain percentage was 8.32 . The short duration cold water soaking caused to increase broken grain percentage in comparison to hot water soaking that proved the accuracy of predicted data.

\section{Validation of the model for changing of head rice yield percentage}

Figure 3 shows the actual and predicted values of head rice yield percentage with different parboiling treatment. It was clear from the Figure 3 that predicted head rive yield has varied by similar pattern with actual head rice yield. The goodness of fit of actual values with predicted values of head rice yield percentage was 7.92. In this study, degree of milling and rice variety was kept constant for all parboiling treatment combinations. Hence, low broken grain results for high head rice yield. Chattopadhyay and Kunze [12] reported that reduction of grain breakage during milling operation and increasing of head rice yield is due to sufficient time soaking and steaming.

\section{Validation of the model for changing of rice kernel hardness}

Figure 4 shows the predicted and actual values of rice kernel hardness with parboiling treatments. The results pointed out that predicted rice kernel hardness values were very similar to the actual values because goodness of fit of actual values with predicted values was reported 6.73. Following researchers reported similar results that changed of rice kernel hardness with parboiling treatments. Mohandass [13] reported un-parboiled rice (raw rice) was soft and the parboiled rice hard; the hardness increasing with the duration of soaking steaming and steaming pressure. Islam et al. [14] reported soaking duration, paddy soaked water temperature and steaming pressure mainly responsible for the hardness (textural quality) of parboiled rice.

\section{Goodness of fit of the mathematical model}

Actual values of rice kernel whiteness, broken grain percentage, head rice yield and rice kernel hardness under the different parboiling treatment was very similar to the predicted values obtained by the mathematical model. The average value of above goodness of fit (E) values was reported 7.22. This average value (7.22) was considered as a goodness of fit of this mathematical model. Goodness of fit value (7.22) is less than 10 hence, the mathematical model is indicative of responsibility good fit. Hence, it can be concluded that the resulted mathematical model is good fit (suitable) to predict the values similar to actual values.

\section{Conclusion}

Goodness of fit of this resulted mathematical model (7.22) is less than 10. Hence, it is revealed, that the mathematical model is capable to predict values similar to actual values. The mathematical model was suitable and accurate for obtaining the rice quality values such as rice, broken grain percentage, head rice yield percentage, rice kernel whiteness and hardness by the function of water temperature and time of soaking in paddy parboiling. Hence, mathematical model can be successfully adopted to overcome drawbacks of parboiling process such as loss of colour and hardness (texture) by optimizing the parboiling treatments according to the consumer requirement. It is also useful to minimizing parboiling cost in rice processing. Finally it can be concluded that the mathematical model for rice quality simulation by the function of paddy parboiling treatments was good to fit for BG 358 short grain rice variety.

\section{References}

1. Bhattacharya KR (1985) Parboiling of rice. Rice, Chemistry and Technology American Association of Cereal Chemists.

2. Pillaiyar $P$ (1988) Parboiling. Rice-post production manual. Wiley Eastern Ltd New Delhi, India.

3. Gariboldi F (1972) Parboiled rice. Rice, Chemistry and Technology. American Association of Cereal Chemists.

4. Gunathilake, DMCC (2009) Development of a Soft Parboiling System for Basmati Paddy, Punjab Agricultural University, Ludhiana, India.

5. Engels C, Hendrickx M, Samblanx S, Gryze I, Tobback P (1986) Modeling water diffusion during long-grain rice soaking. J Food Eng 5: 55-73. 
Citation: Gunathialke DMCC (2018) Validation of Parboiling Rice Quality Simulation Mathematical Model. J Food Process Technol 9: 746. doi: 10.4172/2157-7110.1000746

Page 5 of 5

6. Bakshi AS, Singh RP (1980) Kinetics of water diffusion and starch gelatinization during rice parboiling. J Food Sci 45: 1387-1392.

7. Crank J (1975) The mathematics of diffusion. Oxford University Press Inc, New York.

8. Bellow MO, Tolaba MP, Suarez C (2005) Water absorption and starch gelatinization in whole rice grain during soaking. LWT Food Sci Technol 40: 313-318.

9. Bal S (1974) Measurement of milling quality of paddy. REPC Publication.

10. AOAC (2000) Official methods of analysis. (17th edtn), Gaithersburg, MD, USA.
11. Gariboldi F (1974) Rice processing. Food and Agricultural Organization of the United Nation, Rome Italy.

12. Chattopadhyay PK, Kunze OR (1986) Fissuring characteristics of parboiled and raw milled rice. Trans ASAE 29: 1760-1766.

13. Mohandass R, Pillaiyar $P$ (1980) An extrusion test for determining the palatability of parboiled rice. J Food Sci Technol 17: 244-246.

14. Islam MR, Shimizu N, Kimura T (2003) Energy requirement in parboiling and its relationship to some important quality indicators. J Food Eng 63: 433-439. 